\title{
Based on LabVIEW and Multisim Virtual Electronic Platform in Practice Teaching
}

\author{
Lei Wang \\ Department of Information Engineering, Tangshan College, Tangshan, Hebei, China
}

\begin{abstract}
With the development of technology in the field of virtual instrument and network, the undergraduate colleges in different regions have begun to pay more and more attention to the application of electric simulation system in teaching. This paper designs a virtual electronic platform by node-analysis method and transfer method with LabVIEW and Multisim. This system is designed with user login module and the simulation module and implements the user ID authentication and remote simulation. It has been proved to be stable, enhancing the students learning efficiency and practical ability.
\end{abstract}

Keywords - LabVIEW, Multisim, electronic platform, simulation

\section{基于 LabVIEW 和 Multisim 虚拟电子教学平台在实践教 学中的应用}

\author{
王蕾
}

1)唐山学院信息工程系, 唐山市, 河北省, 中国

摘 要 随着虚拟仪器技术和计算机网络技术的发展, 地方型本科院校逐渐重视电子仿真系统在实践教学中的应用。本文利用 LabVIEW 和 Multisim 软件, 通过结点法和调用法设计了虚拟电子实践平台。该系统具有用户登录模块和仿真模块, 实现了用户身份 认证和远程仿真功能。经测试, 系统运行稳定, 提高了学生学习效率, 培养了学生实践动手能力。

关键词 LabVIEW, Multisim, 电子教学平台, 仿真

\section{1. 引言}

电子类基础课程是工科各专业的重要专业基础课, 意 在帮助学生在本科阶段系统建立电子工程思维模型, 同时 也为后续专业核心课程缔造坚实基础, 该类课程重点培养 学生动手实践能力和工程应用能力[1]。随着国内高校纷纷 加入 MOOC (慕课)计划, 越来越多的大学生成为 MOOC 学习者。经初步研究显示, 清华、北大等一批知名高校于 2013 年加入 edX , 其中《电路原理》、《电子线路》课程已 经上线对外开放, 但教学内容大部分都集中在理论探讨方 面, 缺乏对实践层面的聚焦与关注。地方型本科院校在电 子类实践课程教学中多采用传统的现场演示教学, 学生把 主要精力放在电路连接和实验设备调试上, 而由于时间关 系学生对实验创新不足。

我院是一所以工为主的多学科协调发展的普通本科院 校。在近年来的课程调整中, 电子类理论学时不断压缩,
而实践学时比例增加, 如何更好地补充理论教学内容, 充 分调到学生自主学习兴趣是实践改革的重点。电路实验学 时一般占总学时的 $13 \%$ 25\%, 电子电路实验室主要承担 本院 4 个工科系开出的专业基础通识课程的实验指导工 作, 包括针对 4 个电类专业的电路原理实验课程和 10 个非 电类专业的电工学、电工技术实验课程。在 2014 学年, 实 验室共指导学生实验近万人次。调研显示: 依赖于传统教 学方式的电路实验教学工作量大, 学生自主学习能力不强, 实验效果不理想; 实验课程教学内容联系工程应用背景较 少, 教师多采用填鸭式教学方式, 学生学习兴趣不高; 实 验教学系统性不强, 学生对数据来源、调试问题和数据总 结存在较多疑问。

随着计算机技术、信号处理技术和虚拟仪器技术高速 发展, 利用美国 NI 公司的虚拟仪器开发平台一 LabVIEW 和 Multisim 软件设计开发的虚拟仪器实践教学平台可用于 
电工学、电子技术和相关课程设计的实践教学 [2]。 LabVIEW 作为虚拟实验开发系统的代表, 可以利用 Internet 进行虚拟实验室的网络发布, 实现了资源共享, 避免了仪 器重复添置, 满足了学生不再受时间、地点限制进行远程 实验, 开放式的实践平台培养了学生工程思维方式, 锻炼 学生的动手能力和创新能力[3]。

\section{2. 平台实现方案}

本系统实现了基于 LabVIEW 和 Multisim 的虚拟电子 实践教学功能, 使软硬件资源相结合, 在软件界面上观察 电路实验现象, 并实现对用户进行有效管理。系统包括主 要模块:

用户登陆界面设计。其中包括了用户管理界面, 可以 进行增加用户、删除用户和修改密码等操作, 但是在删除 用户中不可以删除管理员用户。在密码验证时, 当错误输 入三次密码时, 系统将自动退出用户界面, 用户必须再次 打开软件进行验证。

虚拟实验部分设计。本平台应用 Multisim 与 LabVIEW 两款软件搭配使用。应用结点法和调用发分别实现系统设 计。首先进行 Multisim 下的软件仿真, 调试成功后进行相 应的设置并保存。然后打开 LabVIEW, 按照实验要求及目 的设置其前面板, 以做到与实验者的友好交互。最后将保 存的 Multisim 文件导入其中, 进行相应操作即可得到实验 室系统下的虚拟实验。平台设计了电子类基础实验、综合 性和创新性实验。

虚拟实践界面设计。新建 VI, 在前界面上设计虚拟实 践的总体界面，本着方便实验者的原则进行设计。

\section{3. 用户登录界面设计}

在虚拟实验室的开发过程中, 主要用到 LabVIEW 提供 的三个模板进行开发。这三个模板是: 工具模板、控制模 板和功能模板[4]。从 VI 层次结构可以看出该模块中有 8 个层次, 而且包含多个子 VI。模块采用由主到次和由表及 里的方式说明。首先看主程序框图如图 1 所示, 大的框架 是有一个 while 循环和一个事件结构组成, 即组建前面板 上可能发生的各个事件。

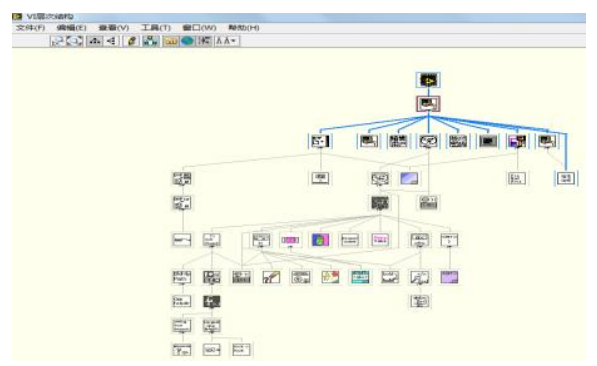

图 1 登录系统结构层次图
此用户登录系统采用 LabSQL 与 Access 数据库进行连 接, LabSQL 支持 Windows 操作系统中任何基于 OBDC 的 数据库, 包括 Access, SQL Server, Oracle, Pervasive, Sybase 等。LabSQL 利用 Microsoft ADO 对象和 SQL 语言来完成 数据库访问, 将复杂的底层 ADO 及 SQL 操作封装成一系 列的 LabSQL VIs, 具有简单易用功能。户登录进入用户登 录界面如图 2 所示。

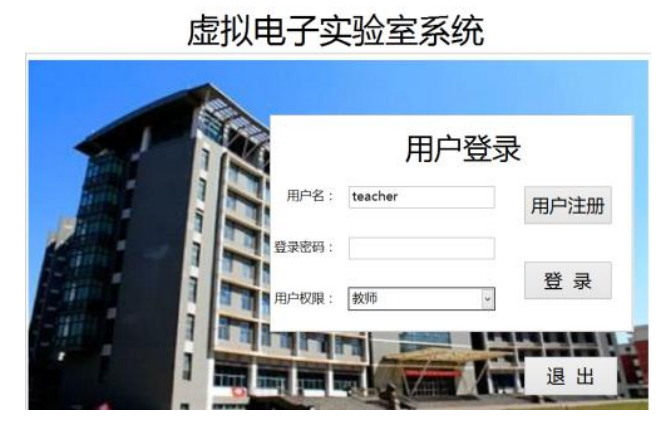

图 2 用户登录系统界面

当用户登录时, 判断用户名和用户密码是否正确和匹 配, 然后在执行相应的程序, 用户权限有教师和学生两种, 教师可以修改权限。其中用了一个子 VI 核对密码, 并显示 用户权限和核对密码是否正确, 调用 3 个条件结构和 1 个 外部条件结构执行密码输入结果相应的程序, 条件假的分 支的内层条件结构执行不同的错误次数的相应程序, 条件 真的内层条件结构用于判断该用户是否是管理员并执行对 应的程序。该程序用到了局部变量和属性节点, 使得程序 实现的功能变得强大, 实现起来也非常简单。用户登录系 统界面后面板，如图 3 所示。

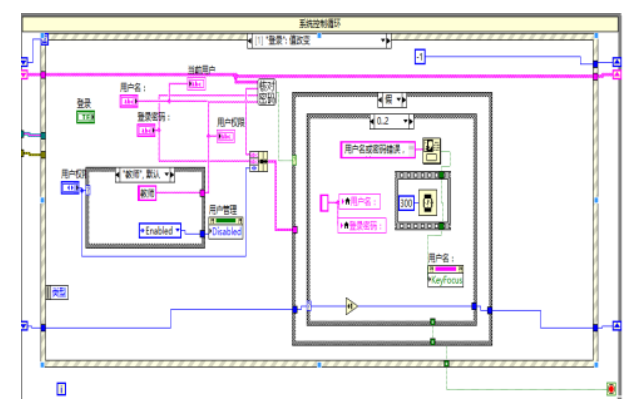

图 3 用户登录系统界面后面板

\section{4. 实践平台的具体实现}

4.1 电路原理设计

该平台设计了多个仿真实例, 现以汽车尾灯控制电路 设计系统为例说明。电路设计要求实现汽车左转弯、右转 
弯、停止等条件下尾灯的点亮与熄灭情况。该系统开关控 制电路由 2 个开关控制, 通过控制对译码电路提供的信号 来控制驱动电路; 三进制计数器电路由 2 个主从 $\mathrm{JK}$ 触发器 构成, 通过主从 JK 触发器的特性构成时序逻辑电路来实现 三进制计数; 译码电路采用 74LS138D 译码器, 使用 3 线 8 线译码器可以控制 8 个端口的输出, 而本实验只需要使用 6 个端口, 其余两个端口闲置。通过三进制计数器和开关 控制电路来控制译码器 6 个端口的逻辑状态; 驱动电路采 用常用的 LED 管, 采用共阳极形式, LED 管的正极接 $+5 \mathrm{~V}$ 电压, 负极通过驱动电路来控制 LED 的亮灭情况。

端口将在 LabVIEW 中以子 VI 的端口承接, 接收和输 出 double 类型的数据流。其端口设置如图 4 所示。

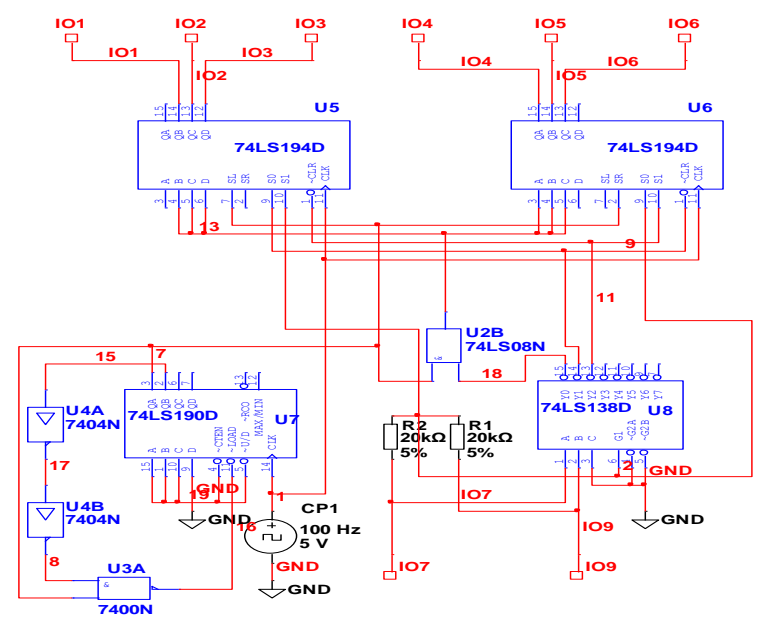

图 4 汽车尾灯加端口设置

设置端口属性 (输入/输出) 将端口的属性设置为对应 的输入 (input) 和输出 (output), IO7 和 $\mathrm{IO} 8$ 为输入量端 口, 分别为左转右转的控件。其中 IO1 为左尾灯 $1, \mathrm{IO} 2$ 为左尾灯 $2, \mathrm{IO} 3$ 为左尾灯 $3, \mathrm{IO} 4$ 为右尾灯 $1, \mathrm{IO} 5$ 为右尾 灯 2, IO6 为右尾灯 3 。

\section{2 利用结点法实现电路设计}

Multisim 与 LabVIEW 建立连接的步骤如下 [5]: 在 Multisim 环境下标记并设置各实验的输入/输出端口; 保存 Multisim 文件在固定的文件夹在下; 现在各模块和所需要 的控件都有了, 现在只需要将控件与调用 Multisim 连接起 来, 组成 LabVIEW 仿真。将各个控件和调用的 Multisim 仿真形成的子 VI 连接起来, 最终组成 LabVIEW 中后面板 仿真图如图 5 所示。这样便可以在运行 LabVIEW 时进行 Multisim 调用、运行。

打开前界面, 按照一定的规则设置前界面, 从功能模
板中找到设计所用的一系列控件。交通灯后面板的设置过 程其主体是 Multisim 与 LabVIEW 的连接过程, 根据原理 分析将各个端口与相应的控件进行连接即可, 结点法后面 板如图 6 所示。

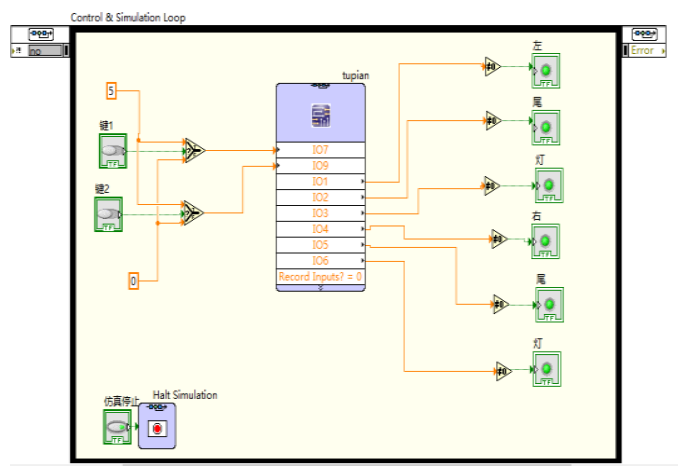

图 5 汽车尾灯背面板

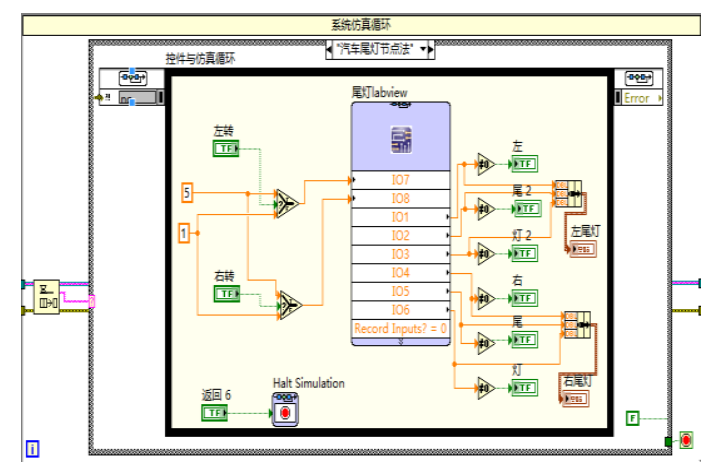

图 6 汽车尾灯结点法后面板图

图 7 为汽车尾灯结点法前界面图中演示的是按下右转 按钮即汽车右转时右边的汽车尾灯亮, 左尾灯不亮, 右侧 左尾灯的示波器没有波形, 右尾灯示波器有脉冲波形所以 右尾灯闪炼, 效果如图 7 所示。

\section{汽车尾灯控制}
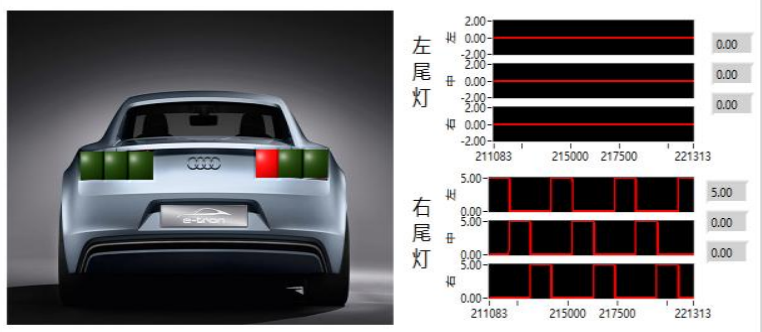

左转右转 提示: 当同时按下

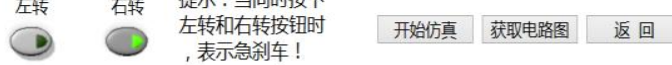

图 7 交通灯右转结点法前界面 


\section{3 利用调用法实现电路设计}

调用法实质是在 LabVIEW 中直接打开 Mutisim, 然后 在 Multisim 中进行电路的仿真。首先点击获取电路图, 即 在 LabVIEW 界面上获得了 Multisim 电路图, 实现这个功 能是利用赋值选项卡。后面板中其实是利用 Multisim Circuit Name 打开文件, 获取了 Multisim 中的电路图, 然 后把电路图转换为二维图片显示到 LabVIEW 前面板中。然 后打开仿真, 找到路径, 通过 $\mathrm{cmd} / \mathrm{x} / \mathrm{c} / \mathrm{start}$ 文件, 找到自 定义路径。最后此虚拟试验系统将在 Multisim 中完成仿真, 如果想回到结点法, 点击返回仿真即可, 页面将自动跳转 到 LabVIEW 仿真中。LabVIEW 与 Multisim 连接的法调用 法实现的虚拟实验仿真的汽车尾灯后面板图, 如图 8 所示。 调用法设计的用户前面板如图 9 所示。

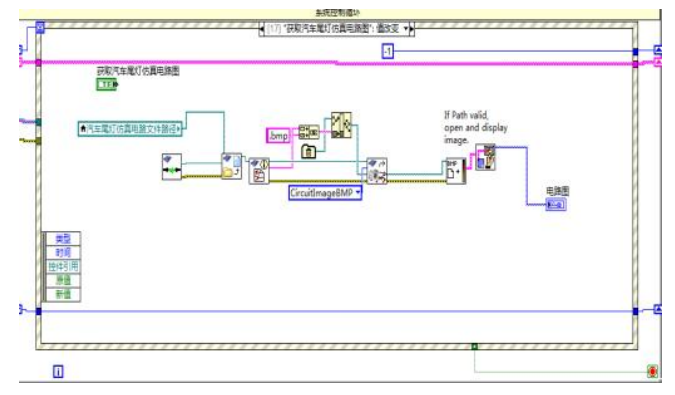

图 8 汽车尾灯调用法后面板图

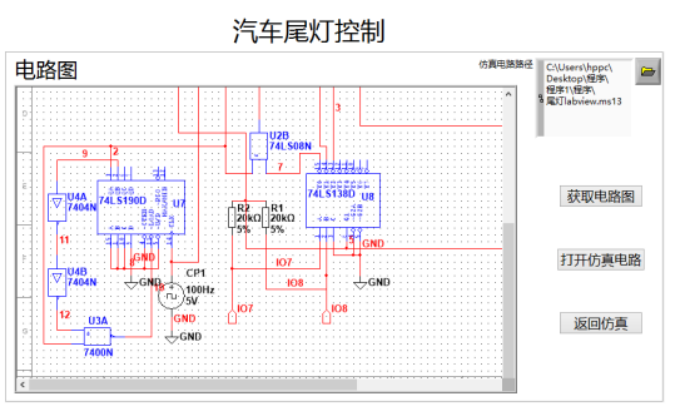

图 9 汽车尾灯调用法前界面

\section{5. 综合调试}

在虚拟实验室系统中每个小实验都要作为一个子 VI 被调用, 所以, 需要将做好的每个小实验进行子 VI 设置。 从功能模板中拖出 while 循环并置于原循环之外, while 循 环为没有循环次数限制的循环。将引用句柄与属性节点进 行连接, 就像程序语言中为一个数据或结构指定了一个具 体的地址, 当执行此 VI 时便由引用句柄将地址指针指向此 VI 保存的地址, 从而实现子 VI 的调用。只有成功设置了 端口才能成功的把 LabVIEW 与 Multisim 连接起来, 程序 才能正常运行。否则, 子 VI 在被调用时无可用端口。同
时 LabVIEW 软件自带了检错, 当有非法的语法错误, 将会有提示错误。如图 10 所示, 汽车尾灯 LabVIEW 仿 真的数据流模式图。

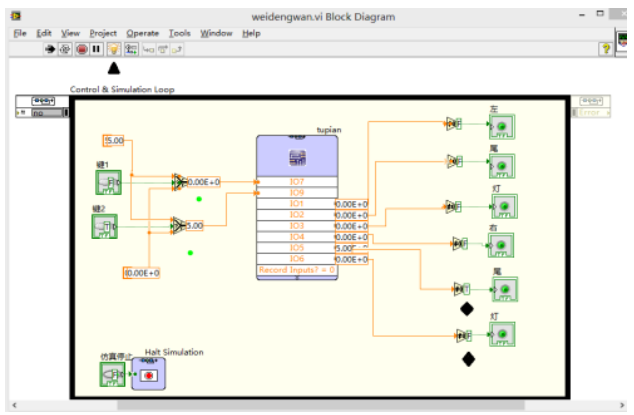

图 10 汽车尾灯数据流图

\section{6. 总结}

传统的实验方式和实验手段往往受制于实验室硬件条 件的制约, 无法满足概念抽象的实验教学要求, 更无法走 出实验室直接参与到课堂教学过程中, 虚拟电子仿真平台 实现彻底改变了这种局面。虚拟实验不但走进了课堂, 而 且改善了教学手段, 改革了教学方式, 使教学效果得到了 极大地提高。本文开发的虚拟电子仿真平台, 充分利用了 LabVIEW 图形化编程的特点和其强大的分析处理功能的 优势，连接 Multisim 仿真软件丰富的电子器件库，使得该 系统非常适合虚拟仿真教学。经过验证，该平台实现真实 实验的演示是完全可行的。电子仿真平台在我院电子类基 础课程的广泛应用, 对于进一步深入开展实践教学的改革 与创新具有一定的指导意义。

\section{参考文献(References)}

[1] Jiahua Mei. The Research of Virtual Experimental Teaching System based on LabVIEW. .Hebei University of Technology. 2012,6-7.

[2] Zhixin Lun. The Design and Realization of Sickbed Calling Device Based on LabVIEW and Multisim. Journal of Tangshan College.2013,71-72.

[3] Cheng Sining, Qiang Geng. Application and Practice of Virtual Simulation Technology in Experimental Teaching of Electrical Courses. Experimental Technology and Management.2013.

[4] Jian Zhang. Desing and Realization on the Teaching Testing System of Virtual Instrumets Based on LabVIEW. 2002,9-10.

[5] Jian Xu.The Research and Development of Experimental Teaching System of Temperature Contronl Based on LabVIEW. 2013,22-26. 